Canadian University Music Review

Revue de musique des universités canadiennes

\title{
Marie-Thérèse Lefebvre. Jean Vallerand et la vie musicale du Québec, 1915-1994. Montréal : Éditions du Méridien, 1996. 109 p. ISBN 2-89415-170-5
}

\section{Johanne Rivest}

Volume 17, numéro 2, 1997

URI : https://id.erudit.org/iderudit/1014796ar

DOI : https://doi.org/10.7202/1014796ar

Aller au sommaire du numéro

Éditeur(s)

Canadian University Music Society / Société de musique des universités canadiennes

ISSN

0710-0353 (imprimé)

2291-2436 (numérique)

Découvrir la revue

Citer ce compte rendu

Rivest, J. (1997). Compte rendu de [Marie-Thérèse Lefebvre. Jean Vallerand et la vie musicale du Québec, 1915-1994. Montréal : Éditions du Méridien, 1996. 109 p. ISBN 2-89415-170-5]. Canadian University Music Review / Revue de musique des universités canadiennes, 17(2), 115-118. https://doi.org/10.7202/1014796ar

All Rights Reserved (C Canadian University Music Society / Société de musique des universités canadiennes, 1997
Ce document est protégé par la loi sur le droit d'auteur. L'utilisation des services d'Érudit (y compris la reproduction) est assujettie à sa politique d'utilisation que vous pouvez consulter en ligne.

https://apropos.erudit.org/fr/usagers/politique-dutilisation/ 
book, but it also obscures the lines of influence that resulted from the cross fertilization of French and Italian traditions. For example, the Neapolitan impresario Domenico Barbaia introduced Italian versions of Spontini's La vestale (1807) in 1811 and Gluck's Iphigénie en Aulide (1774) in 1812. Such was the success of these offerings that when, in 1813, Barbaia offered a contract to Johann Simon Mayr for a new opera on the Medea legend, it stipulated that "the work should be composed in 'the French manner,' which all of us have taken as our model." 4 Thus, I perceive a rather serious lacuna in Kimbell's history of the genre when he attempts to pass from the works of Paisiello to those of Rossini without assessing the influence of the intervening generation of composers simply because they were not resident in Italy.

In spite of the caveats raised above, I believe that Kimbell's study has much to recommend it to both the general and specialist reader, especially since Kimbell's prose is both insightful and entertaining. The book will prove to be useful to university students, although it will need to be supplemented in the areas discussed above. Perhaps the problems of the book could have been avoided had the publisher allowed its author the opportunity to undertake the study in two volumes. Certainly, its scope would have warranted such an expansion into two volumes, even if the rest of the series was restricted to single-volume studies. I hope that Cambridge University Press will give this idea careful consideration. It would be possible then to present a study which could truly embrace all aspects of Italian opera.

Paul F. Rice

Marie-Thérèse Lefebvre. Jean Vallerand et la vie musicale du Québec, 19151994. Montréal : Éditions du Méridien, 1996. 109 p. ISBN 2-89415-170-5.

C'est un fait notoire qu'il existe extrêmement peu de monographies consacrées aux compositeurs québécois récents. Marie-Thérèse Lefebvre contribue à rendre accessible une tranche d'histoire musicale du Québec par cet ouvrage où elle nous introduit à l'une de ses figures importantes, Jean Vallerand. Ce dernier a étudié la composition auprès de Claude Champagne, mais sa formation est avant tout journalistique et littéraire. Il a mené une importante carrière de critique, entre autres aux quotidiens Le Canada, Montréal-Matin, Le Devoir, Nouveau Journal et La Presse. Ce compositeur a également été conférencier, écrivain et animateur radiophonique. Parallèlement, il a occupé divers postes d'administrateur, dont celui de secrétaire général du Conservatoire de musique et d'art dramatique du Québec à Montréal et conseiller culturel auprès de la Délégation générale du Québec à Paris. Il a été professeur d'orchestration au Conservatoire de musique du Québec à Montréal, d'histoire et d'orchestration à la Faculté de musique de l'Université de Montréal, en plus d'avoir dirigé

4Heinrich Bauer, trans. Newell Jenkins and S.W. Bennett, notes accompanying the recording of Mayr's Medea in Corinto (Vanguard VCS-10087/8/9). 
l'exécution de plusieurs œuvres du répertoire. Il a peu composé, mais c'est quand même l'activité qu'on lui reconnaît d'emblée.

Marie-Thérèse Lefebvre entend cependant étudier les aspects musicologiques, critiques et pédagogiques de ce compositeur (p. 13), éléments peu documentés jusqu'à présent (soulignons toutefois l'article de Dominique Olivier, "Jean Vallerand, critique musical ", Les Cahiers de l'ARMuQ 18 [décembre 1996] : 11-24). On connaît l'intérêt de l'auteure pour la pensée des compositeurs québécois importants du $\mathrm{XX}^{\mathrm{e}}$ siècle, telle que véhiculée par leurs écrits. Mentionnons son édition des textes de Garant (Serge Garant et la révolution musicale au Québec [Montréal : Louise Courteau éditrice, 1986], 239 p.) et de Tremblay ("Gilles Tremblay : Réflexions », Circuit, revue nord-américaine de musique du XXe siècle 5, $\mathrm{n}^{\circ} 1$ [1994] : 1-78, et 6, $\mathrm{n}^{\circ} 1$ [1995] : 5-64). Cette fois, Lefebvre ne nous livre pas les textes en entier, ce qui aurait fait l'objet de nombreux volumes, mais en dissémine, au cours du récit, des extraits qui montrent la diversité des champs de compétence et d'intérêt de Vallerand. Leur ordonnance chronologique permet également de saisir l'évolution de certaines de ses idées, dont celles qui sont relatives au sérialisme (les rapports entre Garant et Vallerand sont éloquemment tracés, p. $53,58,71,77,79)$.

L'ouvrage est divisé en trois périodes successives qui correspondent à trois types d'activités de Jean Vallerand : la formation (1915-41, p. 15-30), la carrière musicale (1942-64, p. 31-82) et l'administration (1964-80, p. 83-86). Ces trois chapitres sont d'inégales longueurs; le second, qui constitue la majeure partie de la vie professionnelle de Vallerand, occupe à lui seul presque la moitié de l'étude. Cela s'explique peut-être par le fait que l'ouvrage est largement basé sur les écrits de Vallerand lui-même, et qu'ainsi peu d'information de première source est disponible lorsque Vallerand cesse ses activités de journalisme vers 1964. Six annexes fouillées (p. 92-105) établissent diverses listes d'articles de Vallerand en autant de catégories (articles dans les revues, cours à Radio-Collège, articles sur la responsabilité du critique musical, sur le rôle de l'artiste compositeur, sur la modernité et sur la musique canadienne) et complètent cet essai auquel s'adjoint de plus un index, quoique non exempt d'erreurs, des principaux noms cités.

Au cours de l'étude, l'auteure a le souci de présenter Vallerand dans le contexte politique et idéologique de l'époque au Québec. Elle rend compte avec beaucoup d'à-propos des contradictions de cet « homme de droite quelque peu raciste louchant vers la gauche »(p. 23). Cependant, il me semble que les fondements de la pensée de Vallerand ne se trouvent pas complètement approfondis, surtout parce qu'il manque au travail des points de comparaison qui auraient pu éclairer davantage le sujet. Par exemple, Lefebvre aurait pu opposer Vallerand à Léo-Pol Morin, son prédécesseur immédiat au journal Le Canada, où Vallerand a débuté sa carrière journalistique. De la même façon, les auteurs influents dans le domaine musicologique de l'époque de Vallerand sont répertoriés (p. 41, note 131), mais les aspects de leurs travaux qui ont eu un impact sur les perspectives de Vallerand n'ont pas été vraiment fouillés. Les influences de l'écrivain Georges Duhamel (p. 22, note 33; p. 29) et du chanoine Lionel 
Groulx (p. 30) sont cependant bien présentées. L'aspect le plus intéressant de l'ouvrage est d'avoir montré comment Vallerand apprivoise et épouse peu à peu les objectifs de la modernité ambiante. Lefebvre laisse même entendre que Vallerand aurait été partisan du Refus global, ayant quitté son poste au Montréal-Matin peu après la parution du manifeste pour lequel le rédacteur en chef n'avait que mépris (p. 38). C'est toutefois, selon l'auteure, dans les années 50 que Vallerand se met définitivement "à l'écoute des nouveaux courants de pensée de la modernité » (p. 54). Il explore en effet le sérialisme dans ses propres compositions dès 1955 (p. 77), un an après en avoir dénoncé les limites (p. 53).

Il ne fait pas de doute que Vallerand a été un personnage important, mais il semble que Marie-Thérèse Lefebvre tende à défendre son sujet, ne risquant qu' au compte-gouttes quelques commentaires négatifs (p. 56, note 148). Même si le grand rayonnement des activités de communication de Vallerand rend très probable l'influence qu'il a pu avoir sur des générations de lecteurs et d'auditeurs, l'impact réel de cette influence n'est pas réellement analysé et n'est soutenu que par le témoignage écrit de quelques auditeurs (dont une amie de Vallerand, la cinéaste Anne Claire Poirier, qui signe la préface dans une langue malheureusement fort mal tournée [p. 7-9], et le physicien Hubert Reeves à l'âge de 14 ans [p. 42]). Dans ses cours à Radio-Collège, il semble que la volonté de Vallerand d'instruire, d'apporter des réponses, de poser en éclaireur l'amène à tenir occasionnellement des propos simplistes, garnis de prédictions faciles, comme nous pouvons le constater par ce qui suit.

Où se dirige la musique contemporaine? Vers un classicisme humaniste qui s'affirme de plus en plus dans les œuvres des compositeurs qui s'imposent actuellement à l'attention de l'univers musical.

Quand parviendrons-nous à cet équilibre? Fort prochainement [...]

Pourquoi la musique s'engage-t-elle dans cette voie? Parce qu'elle a épuisé toutes les expériences et qu'elle est maintenant en possession d'un vocabulaire équilibré et discipliné.

Comment cette renaissance s'imposera-t-elle dans le public? Tout normalement par une évolution graduelle. D'ici quelques années, le vocabulaire sera fixé et le public ne sera plus aux prises avec cette incohérence et cette variété de langage qui a créé tant de confusion dans la première partie de notre siècle (p. 52).

Ces propos, amusants pour nous aujourd'hui, trahissaient sans doute à l'époque une certaine pédanterie que l'auteure s'abstient pourtant de dénoncer.

En somme, Marie-Thérèse Lefebvre s'est imprégnée de son sujet, probablement par un élan admiratif justifiable. L'ouvrage acquiert par là les vertus mêmes du sujet en question, c'est-à-dire principalement pédagogiques. L'organisation chronologique et la documentation abondante sur les principaux acteurs sociaux ou sur les tendances des médias de l'époque concernée permettent d'éviter les anachronismes, et donnent au livre la qualité d'un guide historique, voire d'un ouvrage de référence propre à satisfaire étudiants et chercheurs. Mais à ceux qui questionnent malgré tout la pertinence d'un livre sur Vallerand, Lefebvre ne propose que des réponses sommaires, basées sur l'importance que le compositeur tire de l'espace médiatique qu'il a occupé, de 
son intelligence, de ses aptitudes pédagogiques et de sa vaste culture, de même que des bases musicologiques qu'il est censé avoir jetées. Or, nous devons croire sur parole cet honnête verdict, car le « quoi » comparatif manque. En effet, à quelles sources profondes sa pensée s'abreuve-t-elle, quelle en est la portée véritable? Questions auxquelles les solutions font défaut, sans doute parce qu'il y a eu trop peu, à ce jour, d'études de fond sur la musique du Québec contemporain.

À cet égard, l'ouvrage de Marie-Thérèse Lefebvre comble une lacune à laquelle il était plus que temps de remédier. J'aurais souhaité, toutefois, une approche plus critique, pouvant justifier, par exemple, un sous-titre tel que « et la vie musicale du Québec », auquel s'adjoint arbitrairement les dates marquant la naissance et la mort de Vallerand. Même si les opinions de Vallerand sur la musique nous sont révélées dans le livre, la vie musicale comprise entre ces dates n'y est pourtant ni définie ni montrée. J'aurais, pour ma part, préféré un titre plus modeste et proche du sujet, comme "Vallerand vu à travers ses écrits ». Le titre n'enlève cependant rien à la valeur historique et documentaire du livre dont la lecture est un source d'enrichissement appréciable.

Johanne Rivest

Hans Keller. Essays on Music. Edited by Christopher Wintle, with Bayan Northcott and Irene Samuel. Cambridge: Cambridge University Press, 1994. xx, 269 pp. ISBN 0-521-46216-9 (hardcover).

Hans Keller. Three Psychoanalytical Notes on "Peter Grimes." Edited by Christopher Wintle. London: Institute of Advanced Musical Studies in association with The Britten-Pears Library, 1995. 51 pp. ISBN 1-897747-02-0 (softcover).

Hans Keller and Christopher Wintle. Beethoven's String Quartets in F minor, op. 95, and C-sharp minor, op. 131: Two Studies. Nottingham: University of Nottingham, 1995. 55 pp. ISBN 0-9518354-2-4 (softcover).

Hans Keller died on 6 November 1985. A few months later a documentary programme about his life and work was aired on British national television. Tributes were offered by Lord Yehudi Menhuin, Alfred Brendel, Sir William Glock, Robbins Landon, members of the Dartington String Quartet, and prominent individuals from the world of sport and journalism (about which more later). Menuhin talked of Keller's insistence on the highest standards of music criticism; Brendel referred to a public lecture he had once heard Keller give on the Haydn string quartets, delivered extempore ("the finest I have ever witnessed"); while Glock mentioned Keller's well-known ability to read modern scores with fluency. It would have been an impressive enough tribute for a film star. For a musicologist it was unprecedented. 